\title{
FBS framework application based on user requirement analysis
}

\author{
ZHANG Qing, CHEN Deng-kai \\ Shaanxi Engineering Laboratory for Industrial Design, Northwestern Polytechnic University, Xian 710072
}

\begin{abstract}
Users' requirements are key factor for optimizing the design of products. It could be classified into two groups: qualitative research work and quantitative research work. Wireless internet terminal equipment could be used as one example to further study the qualitative research work of requirements. FBS guided by users' requirements was discussed. The users centered service designing process model was established through the analysis of users' needs (Ra) in the function of design, use-pattern, delivery and transformation. Based on this work, the relation between users' needs and requirements of the design was set up. The completely research work about transformation of users' needs to design requirements was discussed in this study, and finally a wireless terminal equipment was used as a case study to verify this method.
\end{abstract}

\section{Introduction}

In the era of large-scale industrial data, with diversified products and complicated functions, the requirements of customers have increasingly become the focus of designs. First of all, industrial design needs to study the requirements of people, and then to solve the problem of how to make products effectively serve users. Industrial design is based on user-centered. The purpose of product design is to improve people's quality of life. Only the designer accurately grasps the user's requirements, the user is likely to buy and have good feedback on the product. User research has long been considered an important stage of design, and it plays an important role in a complete design process. However, the design process deviates from the expected results because subjective factors affect the accuracy of user researches. At present, there are many design methods that can guide user research to play an even greater role. The functionbehavior-structure model (FBS model) was proposed by Gero in 1990. After continuous in-depth research by Gero and other scholars, this model has now formed a complete process of design [1] [2]. This article focuses on how to incorporate user research information into the model so that designers can obtain accurate conclusions that reflect real needs in the process of design.

\section{User research}

Taking wireless Internet access products as an example: When we were doing user research, in order to understand the research object, we selected 100 research subjects and let them describe the concept of wireless internet terminal and choose the products they approve. There are simple and complicated products. Different understanding of product concepts will make different choices. We can get 100 different answers from 100 research subjects, which is mainly because everyone has a different understanding of things. Research subjects need to pick out products that they approve from many electronic products. Basically, they can all find out smoothly. The research object undoubtedly understands the concept of a wireless internet terminal. But their understanding of the concept extension is uncertain. Therefore, we require the research subjects to classify the products according to their shapes, which will cause various deviations. This is part of the research which is difficult to research accurately.

\subsection{Definition of user requirements}

User requirements refer to the user's desire to solve the problem, which are in work or life and cannot be effectively resolved, and need to be resolved by product, technology, or business methods. In terms of the design process, the designer understands what the user really wants through these requirements, so the design will have a direction. But understanding these needs accurately requires a lot of work. Taking a wireless Internet access terminal as an example, the designer should do research to determine whether the user really needs this product and avoid the "detour" in the design. In addition to the normal product background and user questionnaires, we also conducted in-depth analysis of user needs through scene analysis and shooting with follow.

\subsection{Process of user survey}

There are two types of user survey, qualitative research and quantitative research. Qualitative research is an exploratory research with a free form of research, which

*Corresponding author: ${ }^{a}$ zhangqing@e-mail.org 
uses special methods and techniques to investigate the deep information of the target's perceptions, reasons, motivations, etc. Its commonly used methods include user interviews, field survey, usability testing, focus groups, and photo diaries. Quantitative research is a scientific research that investigates the attributes of things and their movement. Accurate data-based research can select more users than qualitative research, analyze big data, and prioritize more important tasks [3]. Figure 1 is the research process of wireless mobile internet terminals, combining qualitative research and quantitative research.

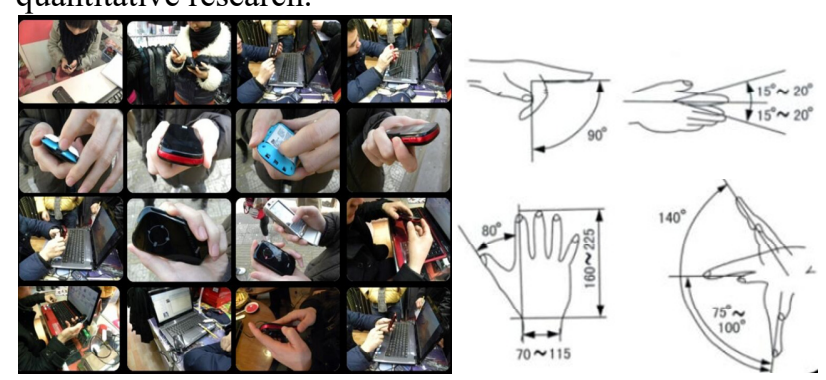

Figure 1. Process of User Survey.

\subsection{Analysis of user requirements}

We need to calculate, analyze semantics and integrate information in order to derive the core requirements from the large amount of information and data obtained from research. The data and information obtained during the research process can be fed back into the design process, as shown in Figure 2

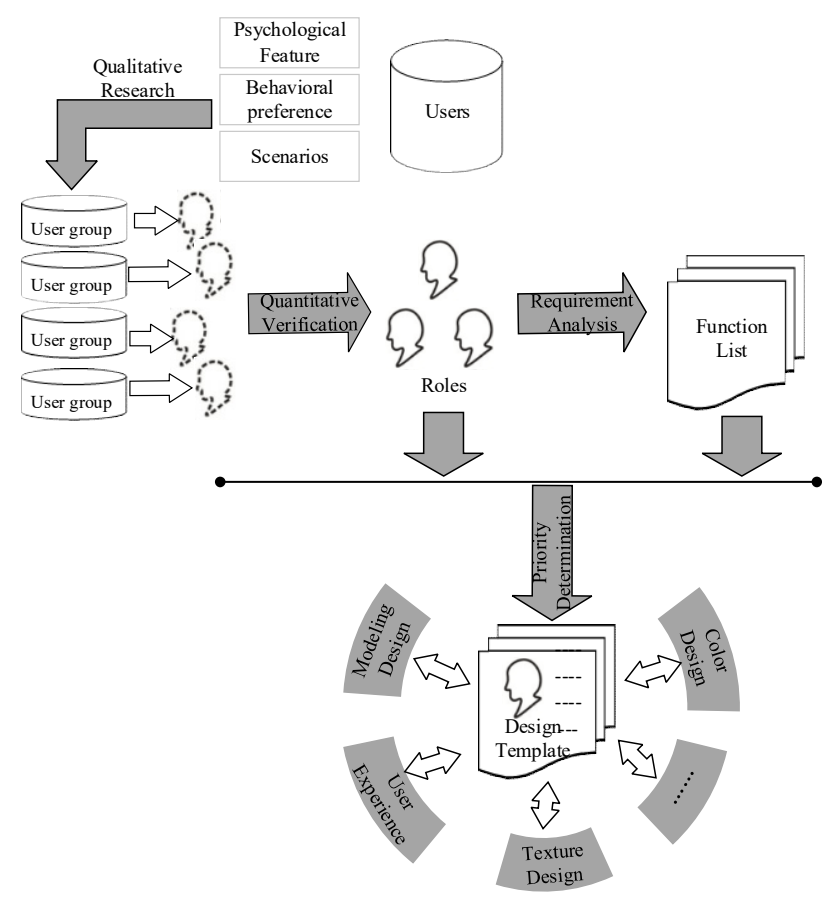

Figure 2. Flow chart of user analysis.

\section{FBS Theory}

The FBS model is a classic model used to describe the process of product design. It transforms the behavioral system (B) to create a corresponding relation between the functional system (F) and the structural system (S). This model contains three variables of function, behavior, and structure, as well as the basic process in the design process. The needs and requirements of the design were originally non-reference variables of the FBS model. However, the needs of users undoubtedly have a dominant role in design in the actual design process. In the previous study, what the first step in the design of any product was designers think about how to translate "function" into "behavior" of product. Gero has proposed a solution to the design in the early stages of FBS theory. That is, four strategies to solve problems that can be used on the basis of the prototype. See Figure 3 .

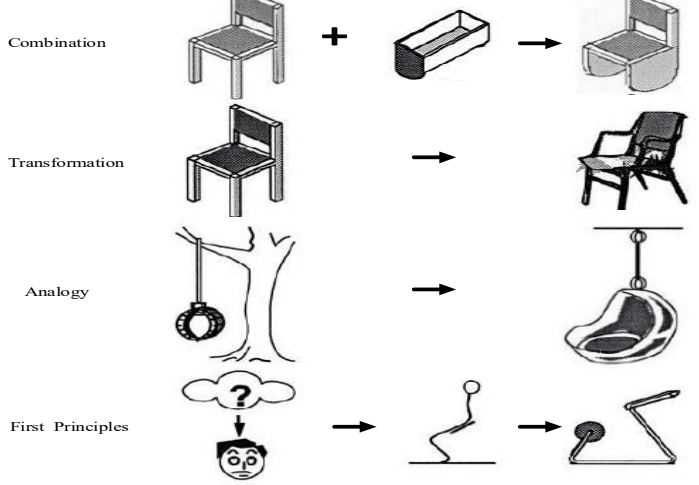

Figure 3. The solution to the design proposed by Gero.

Combination: A recombination or rearrangement of the design scheme. Mutation: This strategy mainly modifies the inadequacy of certain attributes and makes changes to the design scheme. Analogy: This strategy focuses on innovation by selecting similar features and extracting similarities. The first principle: This strategy focuses on getting the proper form and results[4] from the original requirements or functions of the design.

In 2004, Gero continued to refine the FBS model and introduced a new variable: Requirements. This study interprets the world-wide concepts $\mathrm{Fi}, \mathrm{Bi}$, and $\mathrm{i}$ through design models. Therefore, the demand R[5] is also wellinterpreted. By summarizing these model methods and combining Gero's strategy for solving the four problems, we can obtain the other corresponding three strategies:1.In order to extract the key elements of user needs, we can use the strategies of "mutation" or "first principle" to amend or get rid of the unreasonable aspects of the design scheme. It applies to the development stage of the design scheme. We can continue to modify the design to form a collection of design schemes. In the end we will get a satisfactory scheme.2. At the stage of generating the scheme, we need to use the strategy of "combination" to merge all the demand points, thereby motivating new innovations of design.3. The strategy of "analogy" will motivate more innovations. We use this strategy at the generation and development stage of scheme. The strategy of "analogy" can bend the brain in new directions by integrating new elements. In the case of wireless internet terminals, the most numerous design schemes were created by using the strategy of analogy. 


\section{Research on the combination of user requirements and FBS Model}

Based on the cognitive structure of semantic features and strategies for solving problems, we can determine that analysis (Ra) and behavior (B) are key variables in product design issues. According to requirements of design, designers can use the Ra-FBS model to classify complex and decentralized design issues and then analyze the functional characteristics of the results to solve problems. This will help designers make reasonable choices in the design process that follows.

Throughout the whole design process, the designer first needs to transform user requirements into analysis of user requirements ( $\mathrm{R}-\mathrm{Ra})$ and transform the product functions into expected behaviors that can achieve the functions. Expected behavior can be a structural scheme through requirements' analysis of design strategy. The designer then obtains structure-driven behavior from the structural scheme, and then makes comparisons and evaluations. If the evaluated scheme is incomplete, the designer can repeat the above steps, which can make the step of transforming requirements into functions more clear. When designers analyze user requirements, they can use this method, overall structure, details of simple mechanical structure, calculation of similarity of space conversion function, variation of functional combination, to transform requirements into structural functions. Designers are not satisfied with the function of the optimized structure scheme and can then modify the function. After the structural scheme is redesigned, the designer can finally obtain a more complete design scheme which is based on the user's needs through the description of the structural scheme. Model see Figure 4.The meaning of each variable in the model is described in Table 1.

Table 1. The meaning of the parameters in the model.

\begin{tabular}{|c|c|}
\hline \multicolumn{2}{|c|}{$\mathrm{R}_{\mathrm{a}}$-FBS Variable Definition } \\
\hline Variable symbol & Definition \\
\hline $\mathrm{R}$ & Requirement \\
\hline $\mathrm{F}$ & Function \\
\hline $\mathrm{A}$ & Analogy \\
\hline $\mathrm{B}_{\mathrm{e}}$ & Expected Behavior \\
\hline $\mathrm{B}_{\mathrm{s}}$ & Behavior Derived from Structure \\
\hline $\mathrm{S}$ & Structure \\
\hline $\mathrm{R}_{\mathrm{a}} \longrightarrow \mathrm{Fser} \mathrm{requirementanalogy} \mathrm{result}^{\longrightarrow}$ & \\
\hline &
\end{tabular}

Figure 4. Ra-FBS model framework.
The transformation from behavior to structure, $\mathrm{Be}-$ $\mathrm{Ra}-\mathrm{S}$, in the FBS model can be seen as a change of turn. It clearly shows the structural changes under the influence of different behavioral patterns. User requirements analysis is a variable in this process. A preliminary program set can be combined and formed by various structural changes. In terms of style, color, and human-computer interaction, we can generate and optimize a set of design schemes by strategies of combining, classifying, and mutating, which will ultimately result in a set of diverse and understandable design schemes.

\section{Real design case about system verification}

With various functions and shapes, the wireless internet terminal products are typical and complex products. The product is designed using the analysis model of user requirements, Ra-FBS. The analysis of different types of requirements is the basis for the development of enterprise products. The designer transforms uncertain user requirements into points in the design, so that the design requirements are clear, resulting in innovative products that have both real consumer needs and product design quality.

The Ra-FBS design process shown in Figure 5 applies to industrial products which are based on requirements analysis. However, in the actual design process, there are many uncertain factors. Because of the expansion of designer's thinking, the design process is not fixed, and some design steps are neglected in different stages. This is directly related to the differentiation of the selected objects, and they can all be flexibly adjusted. The design of the wireless internet terminal equipment adopts the model framework, RaFBS, and the corresponding design process is as follows:

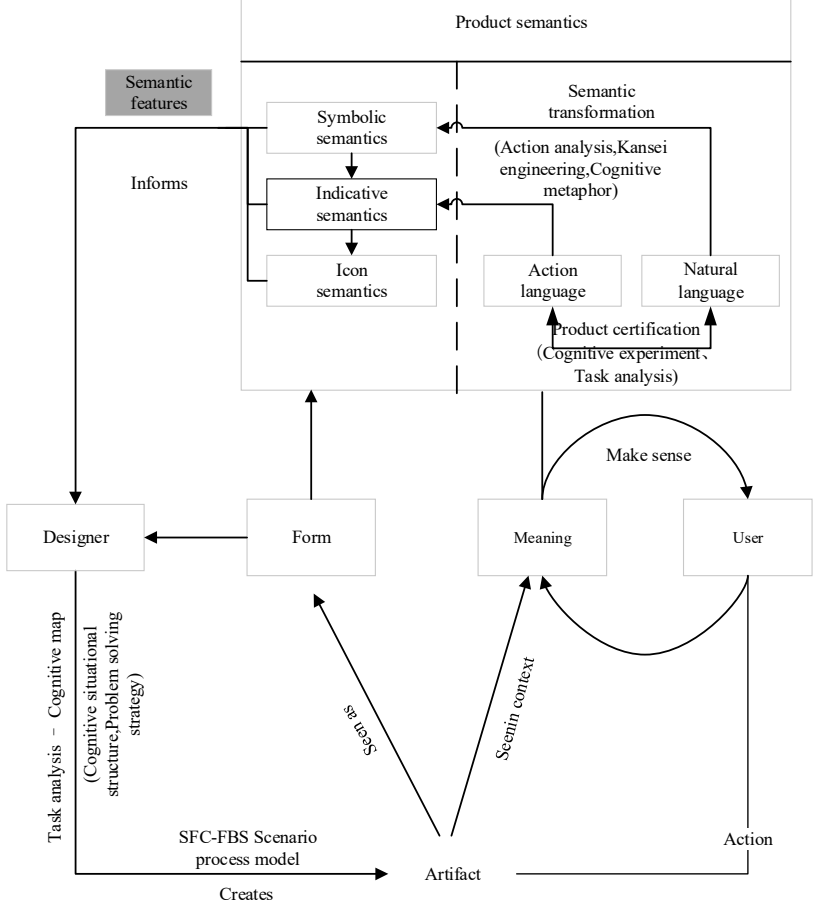

Figure 5. Design process based on Ra-FBS. 
Step 1: $\mathrm{R} \rightarrow \mathrm{Ra} \rightarrow \mathrm{F}$. We transform user requirements into product functions through $\mathrm{Ra}$, and then analyze the qualitative structure and functions of this case to divide the wireless internet terminal equipment into various combinations. The core part of the design is the main function of the product. We analyze the usage to get the product's usage habits. Step 2: $\mathrm{F} \rightarrow \mathrm{Be}$. We transform product functions based on different user requirements into expected behavior, and then compare and analyze similar products. Step 3: $\mathrm{Be} \rightarrow \mathrm{Ra} \rightarrow \mathrm{S}$. Based on the analysis of different user requirements, designers integrate to form a design scheme. We combine product shapes, colors and patterns under the guidance of style features, in the horizontal dimension of the paradigmatic axis. We extract design elements from features and use strategies of analogy and mutation for these elements and pattern features. Finally we got three series with a total of 8 design schemes. Step $4: \mathrm{S} \rightarrow \mathrm{Bs} \rightarrow \mathrm{Be} \rightarrow \mathrm{Ra} \rightarrow \mathrm{S}$. We need to re-evaluate and modify the design i $n$ this step. The step of $\mathrm{S} \rightarrow \mathrm{Bs}$ is to evaluate whether the design scheme satisfies the rationality of the structure through relevant theory. In the step of $\mathrm{Bs} \rightarrow \mathrm{Be}$, the designer compares and evaluates the design scheme and the initial design in order to select a more complete scheme and remodify. In the step of $\mathrm{Be} \rightarrow \mathrm{Ra} \rightarrow \mathrm{S}$, the designer selects the morphological changes of the core product mainly through strategies such as combination, inheritance, and first principles, and finally produces a series of wireless internet terminal products. Figure 6 shows the case.

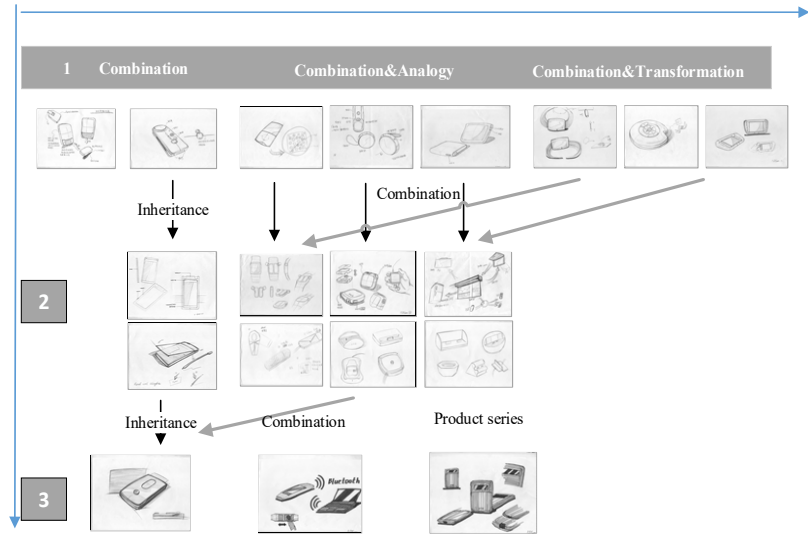

Figure 6. Design process based on Ra-FBS.

\section{Conclusion}

Based on user requirements, this article systematically constructs the function-behavior-structure feedback model (Ra-FBS), which forms a complete method for designing industrial products from user requirements to designers' requirements. With this method, the user's needs are clearly reflected in the actual function of the product in the design process. This article only studies the combination of user requirements and the FBS model, ignoring the impact of other design processes on the product. In further research we should pay attention to the impact of behavior and structure on the product.

\section{References}

1. Gero J S. Design Prototypes: A Knowledge Representation Schema for Design[J]. Ai Magazine, 1990, 11(4):26--36.

2. Gero J S, Kannengiesser U. Representational affordances in design, with examples from analogy making and optimization[J]. Research in Engineering Design, 2012, 23(3):235-249.

3. Galle P. The ontology of Gero's FBS model of designing[J]. Design Studies, 2009, 30(4):321-339.

4. Gero, J. S., Kannengiesser, U. The situated function - behavior - structure framework[J]. Design Studies, 2014, 25(4): 373-391.

5. Gero, J S., Kannengiesser, U. The Function Behaviour - Structure Ontology of Design[M]. An Anthology of Theories and Models of Design. Springer London, 2014:263 -283. 